\title{
BMJ
}

\section{Low energy diet and intracranial pressure in women with idiopathic intracranial hypertension: prospective cohort study}

\author{
Alexandra J Sinclair, MRC research fellow, ${ }^{1,2}$ Michael A Burdon, consultant ophthalmic surgeon, ${ }^{1}$ Peter G \\ Nightingale, trial statistician, ${ }^{3}$ Alexandra K Ball, clinical research fellow neurology, ${ }^{4}$ Peter Good, consultant \\ clinical scientist, ${ }^{5}$ Timothy D Matthews, consultant ophthalmic surgeon, ${ }^{5}$ Andrew Jacks, consultant \\ ophthalmic surgeon, ${ }^{5}$ Mark Lawden, consultant neurologist, ${ }^{6}$ Carl E Clarke, professor of clinical neurology, ${ }^{4}$ \\ Paul M Stewart, director of research and professor of medicine, ${ }^{2}$ Elizabeth A Walker, lecturer in \\ endocrinology, ${ }^{2}$ Jeremy W Tomlinson, MRC senior clinical fellow and consultant in diabetes and \\ endocrinology, ${ }^{2}$ Saaeha Rauz, senior lecturer and consultant ophthalmologist ${ }^{1}$
}

\footnotetext{
'Academic Unit of Ophthalmology, School of Immunity and Infection, College of Medical and Dental

Sciences, University of Birmingham, Birmingham B15 2TT

${ }^{2}$ Centre for Endocrinology, Diabetes and Metabolism, School of Clinical and Experimental Medicine, College of Medical and Dental Sciences, University of Birmingham, Birmingham

${ }^{3}$ Wellcome Trust Clinical Research Facility, College of Medical and Dental Sciences, University of

Birmingham, Birmingham

${ }^{4}$ Neurosciences, School of Clinical and Experimental Medicine, College of Medical and Dental Sciences, University of Birmingham, Birmingham

${ }^{5}$ Birmingham Neuroophthalmology Unit, University Hospitals Birmingham NHS

Foundation Trust, Birmingham B15 2TH

${ }^{6}$ Department of Neurology, Leicester General Hospital, Leicester LE5 4PW

Correspondence to: S Rauz s.rauz@bham.ac.uk
}

Cite this as: $B M J$ 2010;340:c270 doi:10.1136/bmi.c2701 patients, the LogMAR visual acuity improved by one line.

\section{ABSTRACT}

Objective To observe intracranial pressure in women with idiopathic intracranial hypertension who follow a low energy diet.

Design Prospective cohort study.

Setting Outpatient department and the clinical research facility based at two separate hospitals within the United Kingdom.

Participants 25 women with body mass index (BMI) $>25$, with active (papilloedema and intracranial pressure $>25$ $\mathrm{cm} \mathrm{H}_{2} \mathrm{O}$ ), chronic (over three months) idiopathic intracranial hypertension. Women who had undergone surgery to treat idiopathic intracranial hypertension were excluded.

Intervention Stage 1: no new intervention; stage 2: nutritionally complete low energy (calorie) diet (1777 kJ/day (425 kcal/day)); stage 3: follow-up period after the diet. Each stage lasted three months.

Main outcome measure The primary outcome was reduction in intracranial pressure after the diet. Secondary measures included score on headache impact test-6, papilloedema (as measured by ultrasonography of the elevation of the optic disc and diameter of the nerve sheath, together with thickness of the peripapillary retina measured by optical coherence tomography), mean deviation of Humphrey visual field, LogMAR visual acuity, and symptoms. Outcome measures were assessed at baseline and three, six, and nine months. Lumbar puncture, to quantify intracranial pressure, was measured at baseline and three and six months. Results All variables remained stable over stage 1 . During stage 2, there were significant reductions in weight (mean 15.7 (SD 8.0) kg, P 0.001), intracranial pressure (mean 8.0 (SD 4.2) $\mathrm{cm} \mathrm{H}_{2} \mathrm{O}, \mathrm{P}<0.001$ ), score on headache impact test (7.6 (SD 10.1), $P=0.004$ ), and papilloedema (optic disc elevation (mean 0.15 (SD 0.23) $\mathrm{mm}, \mathrm{P}=0.002$ ), diameter of the nerve sheath (mean 0.7 (SD 0.8) $\mathrm{mm}$, $\mathrm{P}=0.004$ ), and thickness of the peripapillary retina (mean
25.7 (SD 36.1) $\mu, P=0.001)$ ). Mean deviation of the Humphrey visual field remained stable, and in only five Fewer women reported symptoms including tinnitus, diplopia, and obscurations ( $10 \vee 4, \mathrm{P}=0.004 ; 7 \vee 0$, $\mathrm{P}=0.008$; and $4 \vee 0, \mathrm{P}=0.025$, respectively). Re-evaluation at three months after the diet showed no significant change in weight $(0.21$ (SD 6.8) kg), and all outcome measures were maintained.

Conclusion Women with idiopathic intracranial hypertension who followed a low energy diet for three months had significantly reduced intracranial pressure compared with pressure measured in the three months before the diet, as well as improved symptoms and reduced papilloedema. These reductions persisted for three months after they stopped the diet.

\section{INTRODUCTION}

Idiopathic intracranial hypertension, also known as benign intracranial hypertension or pseudotumour cerebri, is a condition of unknown cause characterised by raised intracranial pressure and papilloedema. ${ }^{1}$ It typically affects young obese women, causing disabling daily headaches and loss of vision, which is severe and permanent in up to a quarter. ${ }^{2}$ The incidence of idiopathic intracranial hypertension among obese women is 20 per 100000 , and the prevalence is predicted to rise in conjunction with the global epidemic of obesity. ${ }^{3}$

There is insufficient evidence to guide therapeutic strategies, as highlighted in the 2005 Cochrane review. ${ }^{4}$ The condition has a striking association with obesity (greater than 93\%), ${ }^{5}$ and consequently weight loss is often suggested as a treatment. Nevertheless, there has been only one previous prospective weight modifying study, carried out 35 years ago, which observed subjective improvement in the papilloedema in nine patients on a low calorie rice $\operatorname{diet}^{6}$; these 


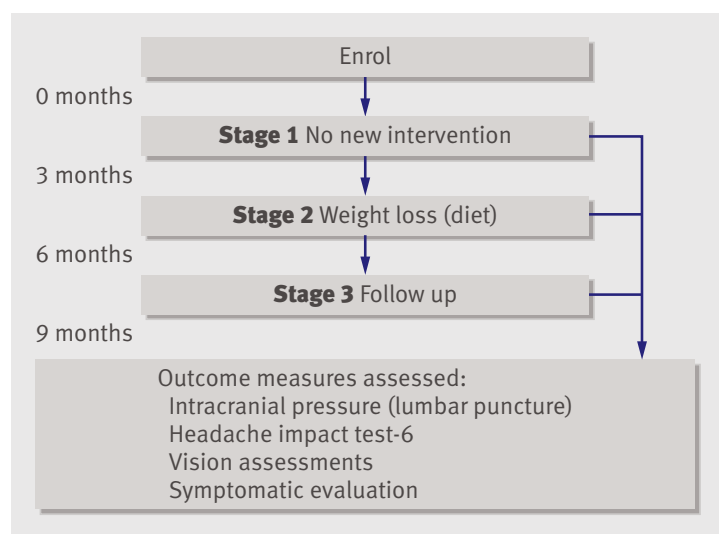

Fig 1| Summary of study design. Vision assessments include measurement of papilloedema by ultrasonographic evaluation of optic disc elevation and nerve sheath diameter as well as optical coherence tomography measurement of retinal nerve fibre layer, LogMAR visual acuity, Humphrey visual field 24-2 mean deviation, Pelli-Robson contrast sensitivity, and Farnsworth-Munsell 100 hue colour assessment. Symptoms evaluated include headache, tinnitus, visual loss, obscurations and diplopia. Lumbar puncture was carried out only at baseline, three and six months

findings were supported by retrospective review of case notes. ${ }^{7-9}$ These studies relied on subjective observation of papilloedema and included people with newly diagnosed idiopathic intracranial hypertension, many of whom improve irrespective of any treatment given. Furthermore, they did not monitor concomitant changes in intracranial pressure with either weight loss or resolution of papilloedema.

We carried out a detailed examination of the interplay between weight loss in patients with idiopathic intracranial hypertension with objective measures of papilloedema and visual function, headache scores and, crucially, changes in intracranial pressure.

\section{METHODS}

\section{Participants}

Women with idiopathic intracranial hypertension were identified and recruited from the Birmingham and Midland Eye Centre, University Hospitals Birmingham NHS Foundation Trust, and University Hospitals of Leicester NHS Trust. Idiopathic intracranial hypertension was diagnosed according to the modified Dandy criteria,${ }^{10}$ with all patients having normal findings on magnetic resonance imaging and venography, papilloedema, intracranial pressure $>25 \mathrm{~cm} \mathrm{H}_{2} \mathrm{O}$, and duration of disease over three months at enrolment. Women were excluded if they had undergone previous cerebrospinal fluid diversion or optic nerve sheath fenestration, were pregnant or planning to conceive, had relevant comorbidity, or were aged less than 16. All women who met the inclusion criteria were invited to take part in the study.

\section{Study design}

Twenty five women were prospectively recruited into a three stage study. Stage 1 was a three month period of no new intervention (control phase); stage 2 comprised a three month intensive diet; and stage 3 was a three month follow-up after completion of the diet (fig 1). Weight loss was achieved with a previously validated, ${ }^{11}$ nutritionally complete, low energy, total meal replacement liquid diet (Lipotrim, Howard Foundation, Cambridge), which provided women with a total of $1777 \mathrm{~kJ} /$ day (425 kcal/day). Patients were advised to consume no additional food, and drink at least 2 litres of fluid a day. During stage 2 all participants underwent weekly review conducted at the Wellcome Trust Clinical Research Facility at the University Hospitals Birmingham NHS Foundation Trust. Compliance with the diet was monitored through evaluation of weight lost and presence of urinary ketones (a feature of abstaining from food intake). Participants were also regularly encouraged, through the provision of weekly nutritional counselling.

Participants were evaluated at baseline and at the end of each stage. Anthropometric data were collected and body mass index (BMI) calculated. The presence of symptoms (tinnitus, visual loss, diplopia, visual obscurations, and headache) together with medication use was recorded. Assessment of visual function under standardised luminescence was carried out with a LogMAR (log of the minimum angle of resolution) chart to assess visual acuity, automated perimetry (Humphrey 24-2 central threshold) to measure the visual field mean deviation, a Pelli-Robson chart to evaluate contrast sensitivity, and a Farnsworth-Munsell 100 hue test to appraise colour vision. (See appendix A on bmj.com for details.)

Papilloedema was evaluated with masked ultrasonography of the optic disc elevation and nerve sheath diameter (maximal retrobulbar pial diameter) by using a high resolution brightness scan (B-scan) with emission frequency $20 \mathrm{MHz}$ and $10 \mathrm{MHz}$, respectively (Quantel Medical, Clermont-Ferrand, France). Additionally, peripapillary retinal nerve fibre layer thickness was measured by optical coherence tomography (Stratus OCT V4.0.1, Carl Zeiss, Meditec, Welwyn Garden City). At each visit a neuro-ophthalmologist carried out masked papilloedema grading of fundus photographs (Frisen rating scale 0 to 5 , the latter being most severe (see appendix B on bmj.com). ${ }^{12}$

Headache phenotype (according to criteria from the International Headache Society ${ }^{13}$ was assessed at baseline. Women completed a daily headache diary in the

Table 1|Baseline demographics in 25 women with idiopathic intracranial hypertension before starting low energy diet

\begin{tabular}{lc}
\hline Mean (SD) age (years) & Data \\
\hline Ethnicity: & $34.4(9.2)$ \\
\hline White & 20 \\
\hline African-Caribbean & 3 \\
\hline South West Asian (Indo-Pakistani) & 2 \\
\hline Mean (SD) duration of disease (months) & $39.0(49.2)$ \\
\hline Taking acetazolamide & 11 \\
\hline
\end{tabular}


Table 2 |Study characteristics and symptom chronology at each assessment. Figures are means (SD) unless stated otherwise

\begin{tabular}{|c|c|c|c|c|c|}
\hline Characteristic & Baseline & Start diet & End diet & Final & Pvalue* \\
\hline Weight (kg) & $101.5(16.0)$ & $102.5(16.8)$ & $86.8(15.6)$ & $87.0(12.9)$ & $<0.001$ \\
\hline BMI & $38.2(5.0)$ & $38.6(5.3)$ & $32.6(4.7)$ & $32.8(4.4)$ & $<0.001$ \\
\hline Intracranial pressure $\left(\mathrm{cm} \mathrm{H}_{2} \mathrm{O}\right)$ & $39.8(5.1)$ & $38.0(5.0)$ & $30.0(4.9)$ & - & $<0.001$ \\
\hline \multicolumn{6}{|l|}{ Vision: } \\
\hline Optic disc elevation (ultrasonography) (mm) & $1.02(0.30)$ & $0.97(0.31)$ & $0.82(0.28)$ & $0.75(0.26)$ & 0.002 \\
\hline Optic nerve sheath diameter (ultrasonography) (mm) & $4.5(1.0)$ & $4.7(1.0)$ & $4.0(1.2)$ & $3.6(1.2) \dagger$ & 0.004 \\
\hline Retinal nerve fibre layer distension (optical coherence tomography) $(\mu \mathrm{m}) \ddagger$ & $144.1(45.5)$ & $135.0(48.0)$ & $109.3(27.9)$ & $108.2(28.6)$ & 0.001 \\
\hline LogMAR visual acuity§ & $-0.02(0.10)$ & $0.01(0.11)$ & $-0.06(0.09)$ & $-0.07(0.08)$ & $<0.001 \dagger$ \\
\hline Pelli-Robson contrast sensitivity§ & $1.58(0.17)$ & $1.61(0.11)$ & $1.67(0.05)$ & $1.68(0.10)$ & 0.003 \\
\hline Humphrey visual field 24-2 mean deviation§ & $-3.8(4.1)$ & $-3.4(3.7)$ & $-2.7(3.0)$ & $-1.5(1.8) \dagger$ & 0.162 \\
\hline Farnsworth-Munsell 100 hue colour assessment $\ddagger$ & $110.7(86.5)$ & $89.3(84.6) \pi$ & $65.7(54.9) \pi$ & $61.3(62.0) \Uparrow$ & 0.027 \\
\hline \multicolumn{6}{|l|}{ Headache: } \\
\hline Impact test (HIT-6)§ & $57.5(9.0)$ & $54.5(1.0)$ & $46.9(10.1)$ & $48.4(9.6)$ & 0.004 \\
\hline Severity (VAS score 0-10)§ & $3.8(2.4)$ & $4.2(2.8)$ & $1.9(2.8)$ & $2.6(2.7)$ & 0.015 \\
\hline Frequency (days/week)§ & $3.8(2.9)$ & $4.4(2.9)$ & $2.1(2.8)$ & $2.6(3.1)$ & 0.011 \\
\hline Analgesic use (days/week)§ & $2.2(2.7)$ & $2.2(2.5)$ & $0.2(0.4)$ & $1.0(2.1)$ & 0.007 \\
\hline \multicolumn{6}{|l|}{ No (\%) with symptoms: } \\
\hline Headache ${ }^{\star \star}$ & $19(95)$ & $19(95)$ & $12(60)$ & $10(50)$ & $<0.001$ \\
\hline Tinnitus** & $10(50)$ & $10(50)$ & $4(20)$ & $4(20)$ & 0.004 \\
\hline Visual loss** & $8(40)$ & $7(35)$ & $3(15)$ & $1(5)$ & 0.063 \\
\hline Obscurations ${ }^{\star *}$ & $3(15)$ & $4(20)$ & $0(0)$ & $0(0)$ & 0.025 \\
\hline Diplopia** & $0(0)$ & $7(35)$ & $0(0)$ & $0(0)$ & 0.008 \\
\hline
\end{tabular}

$\mathrm{BMI}=$ body mass index; LogMAR=log of minimal angle of resolution; HIT-6=headache impact test-6; VAS=visual analogue score.

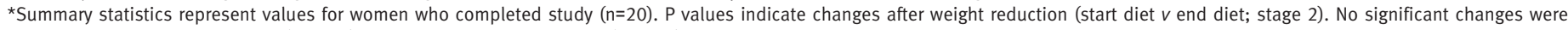
noted from baseline to start diet (stage 1) or from end diet to final visit (stage 3) unless indicated.

†Significant improvement noted but not found in left eye.

$\ddagger$ Data logged before analysis.

$\S$ Friedman's test used for analysis. If result was significant, Wilcoxon sign rank test subsequently carried out, results of latter quoted in table.

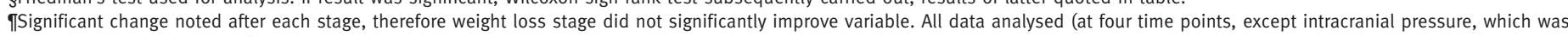
analysed at three time points) with repeated measures analysis of variance unless stated otherwise.

${ }^{\star *}$ Cochran's $\mathrm{Q}$ test showed significant improvement in all variables; sign test subsequently used to evaluate differences at each study stage.

week before each visit, in which they evaluated severity with a visual analogue pain scale (0-10), headache frequency (days/week), and use of analgesia (days/week). The women also completed a headache questionnaire (headache impact test- 6 score range 36-78). ${ }^{14}$

Intracranial pressure was measured by lumbar puncture at baseline and at the end of stages 1 and 2 after all listed outcome measures had been assessed. Measurements were taken with the patient breathing steadily in the left lateral position, legs extended greater than $90^{\circ}$ at the hip, with adequate time taken to ensure a stable reading.

\section{Statistical analysis}

Statistical analysis was performed with SPSS for Windows version 15.0 (SPSS, Chicago, IL) and Prism for Windows version 5.0 (GraphPad Software Inc, San Diego, CA) software packages. Data were reported with means and standard deviations. Serial measurements were analysed with repeated measures: analysis of variance (for parametric or log transformed data) or Friedman's test, with post hoc evaluation achieved with a Wilcoxon signed rank test (for non-parametric data). We analysed dichotomous variables with a Cochran's Q test, and evaluated changes in categorical data with the sign test. Associations between clinical parameters and objective outcome measures were analysed with Pearson's correlation for parametric data and Spearman's rank correlation for non-parametric data. Data were analysed only if present at all time points for each individual. The level at which the results were judged significant was $\mathrm{P}<0.05$ (or $\mathrm{P}<0.017$ for Wilcoxon signed rank test and Sign test as three pairwise comparisons were being made per variable). As data for the right and left eye correlated significantly and yielded analogous results, we have presented only right eye data.

\section{RESULTS}

Of 37 women identified, 25 agreed to take part and 12 declined because of concerns about repeated lumbar puncture. All 37 women had homogeneous characteristics. Among those who agreed to take part, one woman was subsequently excluded as she was found to have a secondary cause of raised intracranial pressure, two did not complete stage 2 of the study as they could not tolerate the diet, and two were lost to followup at the final study visit. Women who withdrew from the study had similar clinical and anthropometric characteristics to those remaining in the study. Table 1 shows participants' demographics. During the study $11(44 \%)$ patients received an unchanging dose of acetazolamide. No other drugs for idiopathic intracranial hypertension were used. There were minimal side 

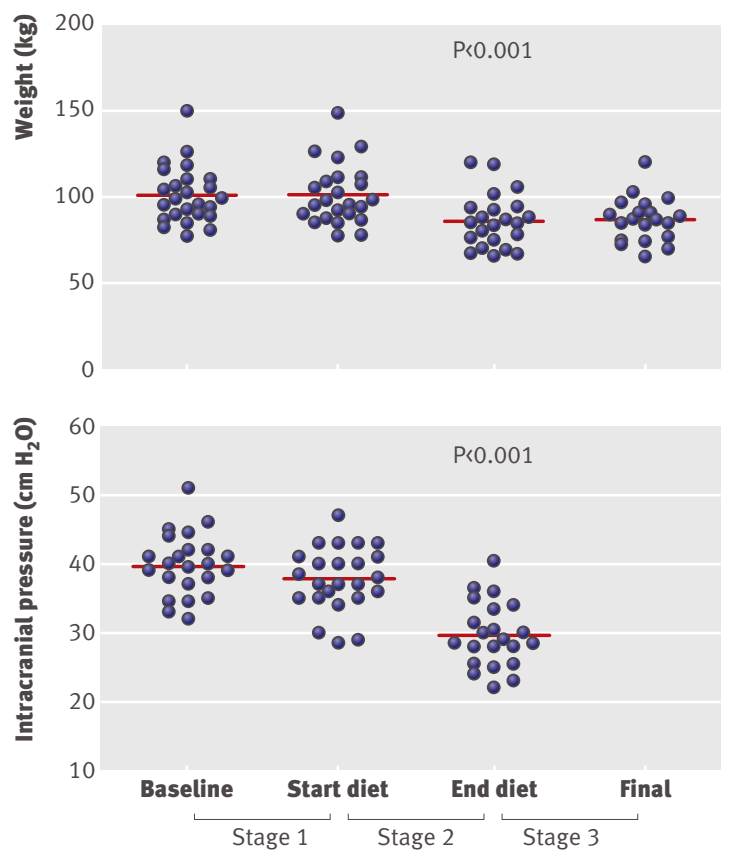

Fig 2 Assessment of change in weight and intracranial pressure

effects associated with the low energy diet: constipation (three patients), light headed feelings (ameliorated by increased fluid intake) (two), and halitosis (one). None of the women underwent diversion of cerebrospinal fluid or fenestration of the optic nerve sheath during the study.

Twenty women completed the entire study and formed the cohort for the analyses. At baseline, mean values were 101.5 (SD 16.0) $\mathrm{kg}$ for weight, 38.2 (5.0) for $\mathrm{BMI}$, and 39.8 (5.1) $\mathrm{cm} \mathrm{H}_{2} \mathrm{O}$ for intracranial pressure (table 2). Universal papilloedema was confirmed with ultrasonography and optical coherence tomography, the most common grade being Frisen grade 2 (16 of 20 cases), with the remainder grade 1 (4 of 20 cases). There was minimal evidence of visual loss, with mean LogMAR acuity of -0.02 (SD 0.10 ) and mean Humphrey visual field mean deviation of -3.8 (SD 4.1) (that is, a low deviation from the variance observed in the general population). Eight women described symptoms of visual loss (such as blurred or distorted vision and blind spots). At the start of the diet three women reported visual obscurations, and 10 reported tinnitus. Seven reported diplopia at the beginning of stage 2 . Headache was almost universally reported (19 of 20 patients) and represented a major source of morbidity (mean headache impact test-6 score 57.5, SD 9.0). Baseline headache phenotyping indicated that 12 out of 20 women had migrainous headache (International Headache Society (IHS) classification 1.1, 1.2, and 1.5.1) in addition to headache attributed to idiopathic intracranial hypertension (IHS 7.1.1) and seven described medication overuse. ${ }^{13}$

As expected, none of the measured variables altered significantly during stage 1 (no intervention). After the low energy diet (stage 2), mean weight fell by 15.7
(SD 8.0) $\mathrm{kg}(\mathrm{P}<0.001 ;-15.3 \%$ (SD 7.0\%) of weight) and mean intracranial pressure by $-8.0(\mathrm{SD} 4.2) \mathrm{cm}$ $\mathrm{H}_{2} \mathrm{O}(\mathrm{P}<0.001)$ (fig 2).[f2] Although all women lost weight and reduced their intracranial pressure, the change in pressure during the low energy diet did not correlate with change in weight $(r=0.280, \mathrm{P}=0.209)$ nor with changes in any of the other outcome measures.

Figure 3 shows that all three measurements used to objectively evaluate different aspects of papilloedema significantly improved after weight loss (stage 2): elevation of the optic disc $(\mathrm{P}=0.002)$, diameter of the optic nerve sheath $(\mathrm{P}=0.004)$, and thickness of peripapillary retinal nerve fibre layer $(\mathrm{P}=0.001)$. There was, however, no significant improvement in the less discriminating Frisen grade during stage 1 or 2 . The actual LogMAR visual acuity score improved significantly $(\mathrm{P}<0.001)$, but this equated to an improvement of only one line (0.1) in five women and was not considered clinically relevant. The Pelli-Robson contrast sensitivity score improved significantly after weight loss (stage 1: four patients improved, two deteriorated; stage 2: eight patients improved, one deteriorated; $\mathrm{P}=0.003)$. During the course of the study, we also noted small improvements in Humphrey visual field mean deviation (0.4 (SD 1.6) for stage $1(\mathrm{P}=0.110)$ and 0.7 (SD 2.4) for stage $2(\mathrm{P}=0.162))$ and Farnsworth-Munsell colour assessment (-21.4 (SD 49.7) for stage $1(\mathrm{P}=0.012)$ and -23.6 (SD 45.3) for stage 2 $(\mathrm{P}=0.027))$. This reflects a learning effect in participants and not a response to weight reduction. ${ }^{15}$

Headache (measured by headache impact test- 6 ) significantly improved after weight loss to a score of 46.9 (SD 10.1) ( $\mathrm{P}=0.004)$ (fig 4). Figure 4 also shows significant improvements in headache severity (visual analogue pain score from 4.2 (SD 2.8) to 1.9 (SD 2.8), $\mathrm{P}=0.015$ ), headache frequency (from 4.4 (SD 2.9) to 2.1 (SD 2.8) days a week, $\mathrm{P}=0.011$ ), and weekly use of analgesics (from 2.2 (SD 2.5) to 0.2 (SD 0.4) days a week, $\mathrm{P}=0.007$ ) (table 2). Patients' symptoms (headache, tinnitus, obscurations, and diplopia) showed significant improvement after the low energy diet (stage 2) $(\mathrm{P}<0.001, \mathrm{P}=0.004, \mathrm{P}=0.025$, and $\mathrm{P}=0.008$, respectively) (table 2).

At the final visit three months after the end of the diet (end of stage 3, table 2), all outcome measures including weight $(0.2$ (SD 2.5) $\mathrm{kg}, \mathrm{P}=0.894)$ were unchanged, with the exception of headache symptoms $(\mathrm{P}=0.002)$, papilloedema (nerve sheath diameter -0.4 (SD 0.8) $\mathrm{mm}, \mathrm{P}=0.035$, and disc elevation -0.07 (SD 0.15) $\mathrm{mm}, \mathrm{P}=0.059$ ), and mean deviation of the Humphrey visual field (1.2 (SD 2.0), $\mathrm{P}=0.009$ ), which continued to improve.

\section{DISCUSSION}

In this small prospective study, women with idiopathic intracranial hypertension who followed a low energy (calorie) diet for three months had significantly reduced intracranial pressure compared with pressure measured in the three months before the diet (headaches and papilloedema also significantly improved). In the three months after the diet ended, weight loss 

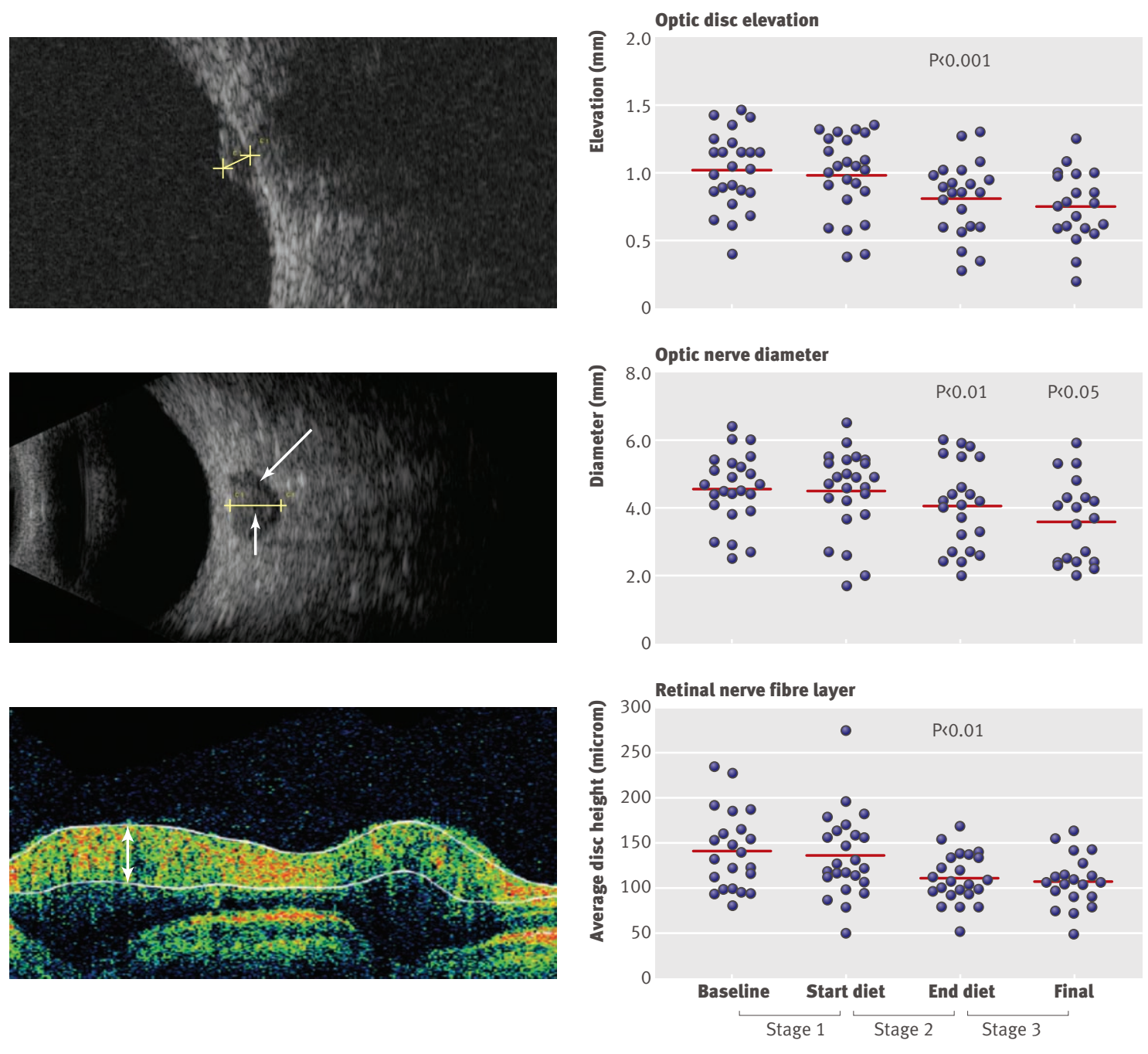

Fig 3 Quantification of papilloedema. Optic disc elevation: in ultrasound image, callipers measure from maximum disc height to lamina cribrosa; scatter plot shows means. Optic nerve diameter: in ultrasound image, callipers mark maximum pial diameter, short arrow indicates cross section through optic nerve, long arrow marks distended cerebrospinal fluid; scatter plot shows means. Retinal nerve fibre layer: in optical coherence tomogram image, arrow marks peripapillary distension; scatter plot shows means. $\mathrm{P}$ values are for changes from previous time point

was maintained and improvements in headache, papilloedema, and vision persisted. These findings suggest that weight reducing diets can help in the management of idiopathic intracranial hypertension.

In four of the 20 women, intracranial pressure fell to a "normal" level (below $25 \mathrm{~cm} \mathrm{H}_{2} \mathrm{O}$ ). In the 16 women whose intracranial pressure remained above $25 \mathrm{~cm}$ $\mathrm{H}_{2} \mathrm{O}, 13$ became asymptomatic for tinnitus, visual loss, diplopia, and visual obscurations after weight loss, compared with four at baseline. These data highlight that it might not be necessary for people with idiopathic intracranial hypertension to reduce their intracranial pressure to below defined normal levels to achieve measurable symptomatic relief. This might, however, be an overly simplistic concept as obesity is known to marginally increase intracranial pressure, ${ }^{16}$ and the equilibrium of cerebrospinal fluid dynamics in resolving idiopathic intracranial hypertension might be re-set such that apparent normality occurs at higher pressures. There was also an absence of a direct relation between weight loss and change in intracranial pressure, potentially suggesting a more complex relation, which could be influenced by a weight threshold for raised intracranial pressure in individual patients.

\section{Measured variables}

Papilloedema, objectively measured with optical coherence tomography, ultrasonography of optic disc elevation, and optic nerve sheath diameter, as well as symptoms (headache, tinnitus, obscurations, and diplopia), all significantly improved after the low energy diet. Papilloedema continued to resolve during stage 3 , despite weight remaining stable. This probably reflects a relatively slow resolution of optic disc axonal distension and oedema after reduction in intracranial pressure. Delayed resolution of papilloedema is also noted after normalisation of intracranial pressure after cerebrospinal fluid shunting for idiopathic intracranial hypertension (that is, the papilloedema resolves 

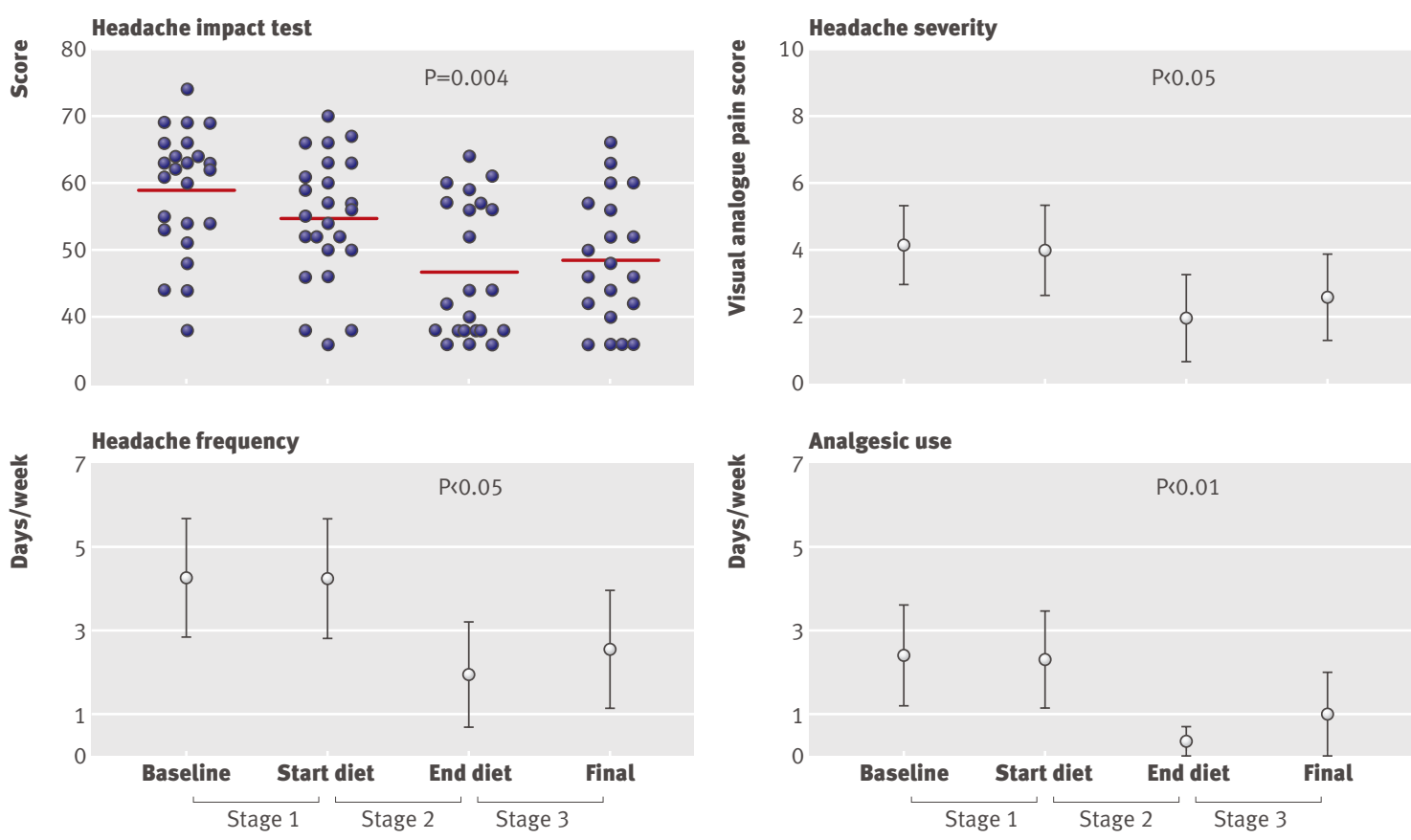

Fig 4 Headache impact test- 6 scores and headache diary assessment, shown as mean changes ( $95 \%$ confidence intervals) from previous time point in headache severity (visual analogue pain score: $0=$ no pain, $10=$ maximum pain), headache frequency, and use of analgesics

over a period ranging from three weeks to seven months after surgery). ${ }^{17}$ The headache impact score, which was substantial at baseline (57.5 (SD 9.0)), significantly improved after the low energy $\operatorname{diet}(\mathrm{P}=0.004)$ to a level that had no or little impact on daily life (46.9 (SD 10.1)) (disability quantification from www.heada chetest.com/HIT6translations.html). ${ }^{18}$ A subgroup of women (eight out of 20) continued to report disabling headaches after the diet, characterised by a score of $>50$ on the headache impact test- 6 , despite a reduction in weight and intracranial pressure equivalent to that of the whole study cohort. Alternative headache phenotypes such as migraine could account for the headache morbidity in these women.

The extent of weight reduction (loss of over 15\% of body weight) is important as numerous other weight loss strategies had previously failed in these women. The low energy diet entailed consumption of three low energy nutrition drinks a day, which varied in flavour. Compliance and consequent universal effectiveness of the diet reflected patients' satisfaction with the regime. This was attributed to the individual weekly review sessions; weekly evidence of successful weight loss, a dependable consequence of consuming only $1777 \mathrm{~kJ} /$ day (425 kcal/day), and a powerful motivating factor; and ketosis, which enhanced hypothalamic satiety, reducing hunger and food cravings once the diet was established. The struggle for patients to achieve and maintain weight reduction however, is, universally recognised. Weight loss through low energy diets, gradual energy restriction, or exclusion of macronutrients (fat, carbohydrates, or protein) could be a little as $2-4 \mathrm{~kg}$ at two years. ${ }^{19}$ Other non-dietary strategies to encourage weight loss include pharmacological and surgical interventions. Pharmacological treatment for obesity, despite tolerability, is unlikely to achieve sufficient weight loss to greatly modify idiopathic intracranial hypertension. After 12 months' treatment, mean weight loss is $2.89 \mathrm{~kg}$ with orlistat ${ }^{20}$ and $4.45 \mathrm{~kg}$ with sibutramine. ${ }^{21}$ Additionally, after concerns about cardiovascular risk, the European Medicines Agency recently withdrew the prescribing licence for sibutramine and the National Institute for Health and Clinical Excellence (NICE) withdrew recommendations for its use. ${ }^{22}$ Topiramate might be a more useful weight modifying drug (reduces weight by $9.75 \mathrm{~kg}$ at 12 months in patients with idiopathic intracranial hypertension), ${ }^{23}$ with additional potential to reduce cerebrospinal fluid secretion via carbonic anhydrase inhibition ${ }^{24}$ and efficacy in treating coexisting migraine. Bariatric surgery could be effective, and one study reported a resolution of papilloedema in a series of eight patients..$^{25}$ Although the advantage of this approach has previously been limited by unacceptable morbidity, recent studies indicate improved safety and efficacy of laparoscopic bariatric surgery and provide rationale for the reevaluation of this technique in people with idiopathic intracranial hypertension. ${ }^{26}$

\section{Strengths and weaknesses}

This was a small study with only 25 patients, which inevitably leads to limitations in interpretation. Because of the intensity of the low energy diet intervention and the detailed clinical protocol, however, performing a study in large numbers of patients before this prospective cohort study might not have been ethically viable. 


\section{WHAT IS ALREADY KNOWN ON THIS TOPIC}

Over $93 \%$ of patients with idiopathic intracranial hypertension are obese

Weight loss is a commonly advocated treatment for idiopathic intracranial hypertension, but there is little prospective evidence for efficacy

\section{WHAT THIS STUDY ADDS}

Weight reduction in women with idiopathic intracranial hypertension was associated with reduced intracranial pressure (measured by lumbar puncture), papilloedema, and symptoms including headache

Encouraging patients with idiopathic intracranial hypertension to embark on and maintain a weight reducing diet might be helpful

The use of objective imaging measures to quantify papilloedema in idiopathic intracranial hypertension mark an important advance that will enhance clinical care and contribute to design of future clinical trials statistically significant improvements in symptoms and papilloedema.

\section{Comparison with other studies}

The only previous prospective study of therapeutic weight loss in idiopathic intracranial hypertension merely assessed subjective changes in papilloedema and included only nine patients. ${ }^{6}$ We not only used objective measures to quantify changes in papilloedema but additionally observed intracranial pressure in patients after a low energy diet.

\section{Implications and future research}

After a low energy diet, women with idiopathic intracranial hypertension had significantly reduced intracranial pressure, headaches, and papilloedema. Our findings suggest that advising and encouraging such patients to embark on and maintain a weight reducing diet could be of therapeutic benefit. Weight loss clinics could have a role in the management of idiopathic intracranial hypertension, though the long term effectiveness and heath economics of this approach will require critical evaluation in a further study comparing weight reduction using NHS dieticians with specially designated weight reduction clinics for patients with idiopathic intracranial hypertension.

We thank Jonas Brane, medical illustrations technician, Sandwell and West Birmingham Hospitals NHS Trust, for his help with the fundus photography.

Contributors: AJS was responsible for study conception, design, recruitment, analysis, interpretation of the data, and drafting of the article. $M A B$, JT, and PMS were responsible for study conception, design, and interpretation of the data. AKB and CRC, were involved in study design. PGN advised on data analysis. PG conducted the ultrasonography. AJ, TDM, and ML facilitated study recruitment. EAW was involved in study conception, data analysis, manuscript redrafting, and decision to submit the manuscript. SR was responsible for study conception, design and interpretation of the data, manuscript redrafting and decision to submit the manuscript, was principal investigator on funding grants, and is guarantor. All authors critically revised the article.

Independence: The funding bodies and study sponsor (University of Birmingham) had no involvement in the study design, data collection, analysis, data interpretation, report writing or decision to submit the manuscript. The low energy (calorie) diet manufacturers, (Lipotrim, Howard Foundation, Cambridge) had no involvement in funding the study nor study design, data collection, analysis, data interpretation, report writing or decision to submit the manuscript.

Funding: This study was funded by the Vera Down (Neurological Diseases) 2005 and Clark and McMaster (Blinding Diseases) 2005 Awards funded by the British Medical Association, a research and development award 2005 from Sandwell and West Birmingham Hospitals NHS Trust, and by the West Midlands Neuroscience Teaching and Research Fund. AJS is a Brain Trust entry/exit scholar, Ipsen Research Fellow and MRC clinical training fellow (G0601430). The Academic Unit of Ophthalmology is supported by the Birmingham Eye Foundation (Registered (UK) Charity 257549). JWT is a MRC senior clinical fellow. Competing interests: All authors have completed the Unified Competing Interest form at www.icmje.org/coi_disclosure.pdf (available on request from the corresponding author) and declare that (1) no authors have support from companies for the submitted work; (2) none of the authors has relationships with companies that might have an interest in the submitted work in the previous 3 years; (3) their spouses, partners, or children have no financial relationships that may be relevant to the submitted work; and (4) none of the authors has financial interests that may be relevant to the submitted work.

Ethical approval: This study was approved by the Dudley local research ethics committee (06/Q2702/64) and informed written consent was given by all participants.

Data sharing: No additional data available. 
1 Sinclair AJ, Ball AK, Burdon MA, Clarke CE, Stewart PM, Curnow SJ, et al. Exploring the pathogenesis of $\mathrm{IIH}$ : an inflammatory perspective. J Neuroimmunol 2008;201-2:212-20.

2 Corbett JJ, Savino PJ, Thompson HS, Kansu T, Schatz NJ, Orr LS, et al. Visual loss in pseudotumor cerebri. Follow-up of 57 patients from five to 41 years and a profile of 14 patients with permanent severe visual loss. Arch Neurol 1982;39:461-74.

3 WHO. Global database on body mass index. 2010. http://apps.who. int/bmi/index.jsp.

4 Lueck C, Mcllwaine G. Interventions for idiopathic intracranial hypertension. Cochrane Database Syst Rev 2005;3:CD003434.

5 Glueck CJ, Aregawi D, Goldenberg N, Golnik KC, Sieve L, Wang P. Idiopathic intracranial hypertension, polycystic-ovary syndrome, and thrombophilia. J Lab Clin Med 2005;145:72-82.

6 Newborg B. Pseudotumor cerebri treated by rice reduction diet. Arch Intern Med 1974;133:802-7.

7 Kupersmith MJ, Gamell L, Turbin R, Peck V, Spiegel P, Wall M. Effects of weight loss on the course of idiopathic intracranial hypertension in women. Neurology 1998;50:1094-8.

8 Johnson LN, Krohel GB, Madsen RW, March GA Jr. The role of weight loss and acetazolamide in the treatment of idiopathic intracranial hypertension (pseudotumor cerebri). Ophthalmology 1998;105:2313-7.

9 Wong R, Madill SA, Pandey P, Riordan-Eva P. Idiopathic intracranial hypertension: the association between weight loss and the requirement for systemic treatment. BMC Ophthalmol 2007;7:15.

10 Friedman DI, Jacobson DM. Diagnostic criteria for idiopathic intracranial hypertension. Neurology 2002;59:1492-5.

11 Tomlinson JW, Moore JS, Clark PM, Holder G, Shakespeare L, Stewart PM. Weight loss increases 11 beta-hydroxysteroid dehydrogenase type 1 expression in human adipose tissue. J Clin Endocrinol Metab 2004;89:2711-6.

12 Frisen L. Swelling of the optic nerve head: a staging scheme. J Neurol Neurosurg Psychiatry 1982;45:13-8.

13 Evers S. [The new IHS classification. Background and structure]. Schmerz 2004;18:351-6.

14 Kosinski M, Bayliss MS, Bjorner JB, Ware JE Jr, Garber WH, Batenhorst A, et al. A six-item short-form survey for measuring headache impact: the HIT-6. Qual Life Res 2003;12:963-74.

15 Castro DPE, Kawase J, Melo LAS Jr. Learning effect of standard automated perimetry in healthy individuals. Arquivos Brasileiros de Oftalmologia 2008;71:523-8.
16 Whiteley W, Al-Shahi R, Warlow CP, Zeidler M, Lueck CJ. CSF opening pressure: reference interval and the effect of body mass index. Neurology 2006;67:1690-1.

17 Bynke G, Zemack G, Bynke H, Romner B. Ventriculoperitoneal shunting for idiopathic intracranial hypertension. Neurology 2004;63:1314-6.

18 Shin HE, Park JW, Kim Yl, Lee KS. Headache impact test-6 (HIT-6) scores for migraine patients: their relation to disability as measured from a headache diary. J Clin Neurol 2008;4:158-63.

19 Sacks FM, Bray GA, Carey VJ, Smith SR, Ryan DH, Anton SD, et al. Comparison of weight-loss diets with different compositions of fat, protein, and carbohydrates. N Engl J Med 2009;360:859-73.

20 FDA. Orlistat (marketed as Alli and Xenical): early communication about an ongoing safety review. 2009. www.fda.gov/Safety/ MedWatch/Safetylnformation/

SafetyAlertsforHumanMedicalProducts/ucm180025.htm.

21 Li Z, Maglione M, Tu W, Mojica W, Arterburn D, Shugarman LR, et al. Meta-analysis: pharmacologic treatment of obesity. Ann Intern Med 2005;142:532-46.

22 Williams G. Withdrawal of sibutramine in Europe. BMJ 2010;340:c824

23 Çelebisoy N, Gökçay F, Eirin HÖA. Treatment of idiopathic intracranial hypertension: topiramate vs acetazolamide, an open-label study. Acta Neurol Scand 2007;116:322-7.

24 Rosenfeld WE. Topiramate: a review of preclinical, pharmacokinetic, and clinical data. Clin Ther 1997;19:1294-308.

25 Sugerman HJ, Felton WL 3rd, Salvant JB Jr, Sismanis A, Kellum JM Effects of surgically induced weight loss on idiopathic intracranial hypertension in morbid obesity. Neurology 1995;45:1655-9.

26 Flum DR, Belle SH, King WC, Wahed AS, Berk P, Chapman W, et al. Perioperative safety in the longitudinal assessment of bariatric surgery. N Engl J Med 2009;361:445-54.

27 Tamburrelli C, Salgarello T, Caputo CG, Giudiceandrea A, Scullica L. Ultrasonographic evaluation of optic disc swelling: comparison with CSLO in idiopathic intracranial hypertension. Invest Ophthalmol Vis Sci 2000;41:2960-6.

28 Rebolleda G, Munoz-Negrete FJ. Follow-up of mild papilledema in idiopathic intracranial hypertension with optical coherence tomography. Invest Ophthalmol Vis Sci 2009;50:5197-200.

29 Concato J, Shah N, Horwitz RI. Randomized, controlled trials, observational studies, and the hierarchy of research designs. N EnglJ Med 2000;342:1887-92.

Accepted: 13 April 2010 